\title{
Improving the Quality of Life of paralyzed dogs and cats using some devices to maintain the anatomical standing position
}

\author{
Mădălina Elena Henea ${ }^{1}$, Liviu Cătălin Burtan², Eusebiu Viorel Șindilar², Iuliana Mihai' ${ }^{2}$, Mariana Grecu ${ }^{3}$, Carmen \\ Solcan ${ }^{4}$, Gheorghe Solcan ${ }^{*}$
}

${ }^{1}$ Phisiotherapy Unit, Clinics Department, Faculty of Veterinary Medicine, University of Life Sciences, Ion Ionescu de la Brad, 700489 Iasi, Romania; madalina.henea@yahoo.com

2Surgery Unit, Clinics Department, Faculty of Veterinary Medicine, University of Life Sciences, Ion Ionescu de la Brad, 700489 Iasi, Romania; burtan liviu@yahoo.com (L.C.B.); esindilar@yahoo.com (E.V.S.); iuliabogdan2005@yahoo.com (I.M.)

${ }^{3}$ Pharmacy Unit, Preclinics Department, Faculty of Veterinary Medicine, University of Life Sciences, Ion Ionescu de la Brad, 700489 Iasi, Romania; marygrecu@gmail.com

${ }^{4}$ Histology Unit, Preclinics Department, Faculty of Veterinary Medicine, University of Life Sciences, Ion Ionescu de la Brad, 700489 Iasi, Romania; csolcan@uaiasi.ro

Internal Medicine Unit, Clinics Department, Faculty of Veterinary Medicine, University of Life Sciences, Ion Ionescu de la Brad, 700489 Iasi, Romania;

* Correspondence: gsolcan@uaiasi.ro; Tel.: (+40745302684)

Simple Summary: Paralysis caused by different lesions of spinal cord or peripherical nerfs profoundly affect the quality of life of pets, many times they being candidates for euthanasia. Many attempts were made to cure the paralyzed pets and to improve their quality of life, including surgical interventions, antiinflammatory and vitamins, acupuncture and electroacupuncture, physiotherapy etc. Physiotherapy aims to prevent and combat diseases of a neurological, orthopedic or even genetic nature, and the main objectives are to regain muscle mass, balance and normal walking. For paralyzed patients, unable to support their own weight, obtaining and maintaining the standing position is essential. To ensure therapeutic success, various devices and exercises are used. We aimed to develop simple devices, accessible to anyone interested in physiotherapy or who takes care of patients with paralysis. The materials used to obtain these devices were easily obtained, at an affordable price, and subsequently they were rapidly assembled.

Abstract: Paralyzed patients have always been ideal candidates for physiotherapy due to their body's inability to recover on its own. Regardless of the cause that led to the onset of paralysis (whether localized or generalized), physiotherapy helps these patients with devices and methods designed to restore their proper functioning of the body, as well as quality of life. Obtaining the standing position is the main stage in the therapeutic process, being the indispensable element for the entire protocol. The present study focused on the devices we developed over time, adapting them for each patient, depending on the degree of damage they presented and the possible associated pathologies. At the same time, we wanted these devices to be available for any therapist or even owner, being made of common materials, easy to find, but also affordable.

Keywords: paralysis, dogs, cats; quality of life, physiotherapy; recovering standing position

\section{Introduction}

Quality of Life is defined as "an individual's satisfaction with its physical and psychological health, its physical and social environment, and its ability to interact with that environment [1]. Paralysis caused by different lesions of spinal cord or peripherical nerfs profoundly affect all the aspects of QoL. Many attempts were made to cure the 
paralyzed pets and to improve their QoL, including surgical interventions, 50 antiinflammatory [2] and vitamins, acupuncture and electroacupuncture [3], 51 physiotherapy [4-8] etc., many times they being candidates for euthanasia.

Physiotherapy includes all the actions of physical factors on an organism, allowing the development of a treatment plan for a complete recovery, but also for prophylactic purposes. This branch of medicine aims to help the body in a critical threshold by restoring the quality of life. Locomotor, neurological and orthopedic diseases are the target on which physiotherapy is oriented, encouraging the body to regain its functionality. Physiotherapy is a combination of therapeutic methods and techniques that help the body recover when it is not capable to do it itself $[4,8]$.

Regarding veterinary physiotherapy in Romania, this branch is still at the beginning $_{\bar{j}}$ and is gradually developing so that many pathologies of neurological, orthopedic, locomotor, joint, muscular, and also genetic nature can be prevented or even treated. The number of diseases which need to be ameliorated or combated through physiotherapy is large, which proves the importance of applying these techniques in the therapeutic protocol. This study presents the techniques developed in the clinic, being adapted according to the condition of each patient (in this case being monoplegia, paraplegia or tetraplegia), allowing the restoration and maintenance of the anatomical standingposition. Initially, the animals were allowed to adapt and get used to the equipment, the environment, as well as the staff that they would come into contact with during the therapeutic sessions.

\section{Materials and Methods}

The study was carried out at the Faculty of Veterinary Medicine in Iași, at the Physiotherapy service. After conducting a thorough clinical examination of each patient, treatment protocols were established using the methods and devices developed over time. As for the materials, they were chosen precisely in terms of their accessibility. The main materials used to obtain the devices meant to restore the standing position are: metal frames with pulley, stable metal frames, wheeled metal frames, cardboard boxes, scarves, towels, as well as plastic bottle caps.

Manual methods aim to restore muscle mass and strength, balance, physiological movement. The implemented therapeutic exercises involved common activities such as: walking on the exercise band, stairs climbing, dancing (to stimulate the hind limbs) or wheel-barrowing (to stimulate the forelimbs). In addition to these common activities, other techniques such as jogging, walking, pulling and moving weights, and crossing tunnels were implemented. Similarly, the game balls were also very useful, as well as positioning a plastic cap over the foot pad of the healthy limb (opposite of the affected limb), thus obtaining equal support on both limbs and better weight support [7].

A great advantage of manual methods is that they can be easily applied and used by the owner at home as they are based on simple equipment, easy to procure and use.

The physiotherapy protocol must be established based on several factors, and the therapeutic program is developed precisely for the needs of each patient [8, 9]. The treatment program is then expanded after a clinical examination identifies the present conditions, techniques and exercises which play a major role for an effective therapy. At the same time, the exercises are chosen so that the patient is completely safe and will achieve complete recovery. During therapeutic sessions, signs of improvement in the patient's health will indicate the need to change the treatment plan, so that the intensity, duration and frequency of an exercise will be increased or decreased depending on the patient's condition $[8,9]$. 


\section{Results}

\section{9}

3.1. Techniques for obtaining and maintaining the standing position

The most suitable patients for these exercises are those who have lost the ability to maintain an anatomical standing position, making it impossible for them to support their own weight. The objectives of this stage are to reconsolidate muscle mass and endurance, regain proprioception, improve the respiratory function and blood circulation, speed up the healing of decubitus ulcers, and support the patient's psychological state. Orthopedic and neurological disorders are the main types targeted by these exercises [9].

\subsubsection{Total assistance for supporting body weight and adopting the standing position}

is applied to animals that have completely lost their ability to remain in the anatomical position, especially after paralysis (Figure 1.). In this case, total assistance means the support of $75-100 \%$ bore by the therapist. Taking into account the patient's height and weight, the therapist can lift them directly, manually or by using equipment represented by specially adapted towels or harnesses [8, 9]. The devices we made specifically to provide more support and to obtain the anatomical standing position were represented by stable metal frames. These metal frames allow the attachment of harnesses or towels for the correct positioning of patients. Once the anatomical standing position is established, the therapist induces limb movements. Thus, the animal is helped to remember its normal position, supporting its body weight with its feet on the ground (Figure 2).

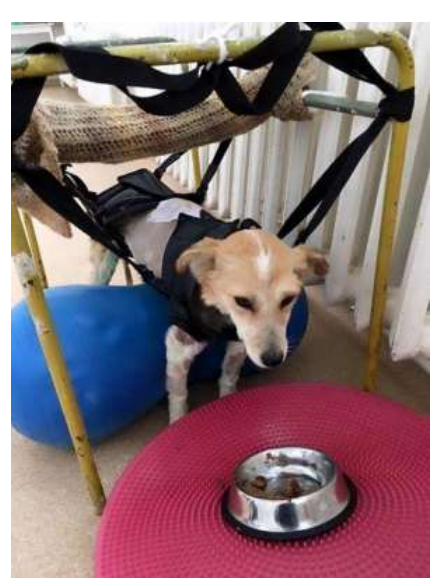

Figure 1. Tetraplegic dog supported in the standing position by harnesses



Figure 2. Paraplegic dog supported in the standing position by harnesses
3.1.2. Standing position actively supported using trolleys and straps.

Trolleys and straps are very useful in physiotherapy sessions (Figure 3). In this regard, in order to obtain a trolley accessible to anyone, we have developed a wheeled metal frame to which a pulley has been added to attach the harness in which the animal is positioned. Thus, the pulley fixed at the top of the metal frame allows the adjustment of the height at which the harness is located and to implicitly induce the standing position of the animal [9].

Trolleys allow animals a sense of freedom, significantly reducing the support provided by the therapist. The two variants of trolleys (with two or four wheels, respectively) are adapted according to the patient's condition: those with two wheels are useful for neuromotor disorders of the hind limbs, while those with four wheels are used in patients with all the four limbs affected. At the same time, the straps are useful for nonambulatory patients (those who are unable to move). For the placement of the straps, the height of the animal will be taken into account for positioning the limbs (performed 
manually by the therapist or automatically, physiologically by the animal) in a natural 134 posture to support body weight (Figures 4 and 5). The animal should not be left hanging 135 above the ground as it may affect local blood supply and press areas directly in contact 136 with the support devices attached to the trolley [10].

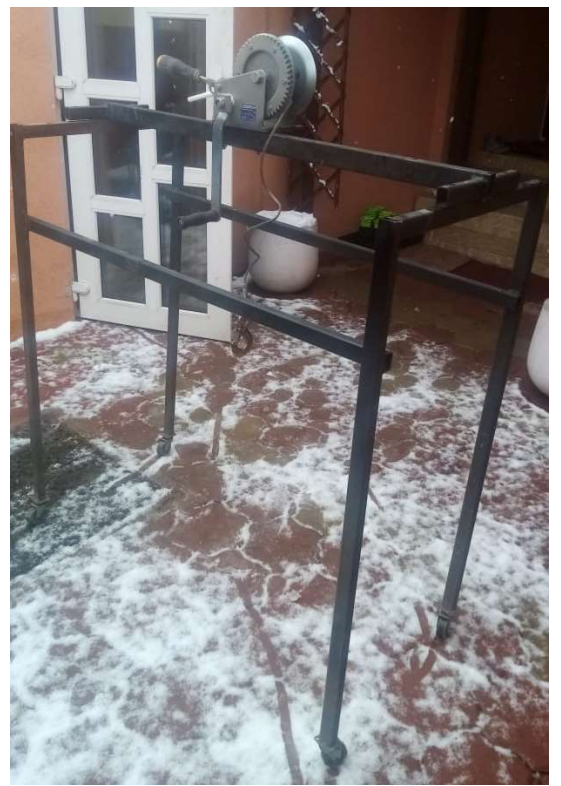

Figure 3. Wheeled device used for implementing the standing position
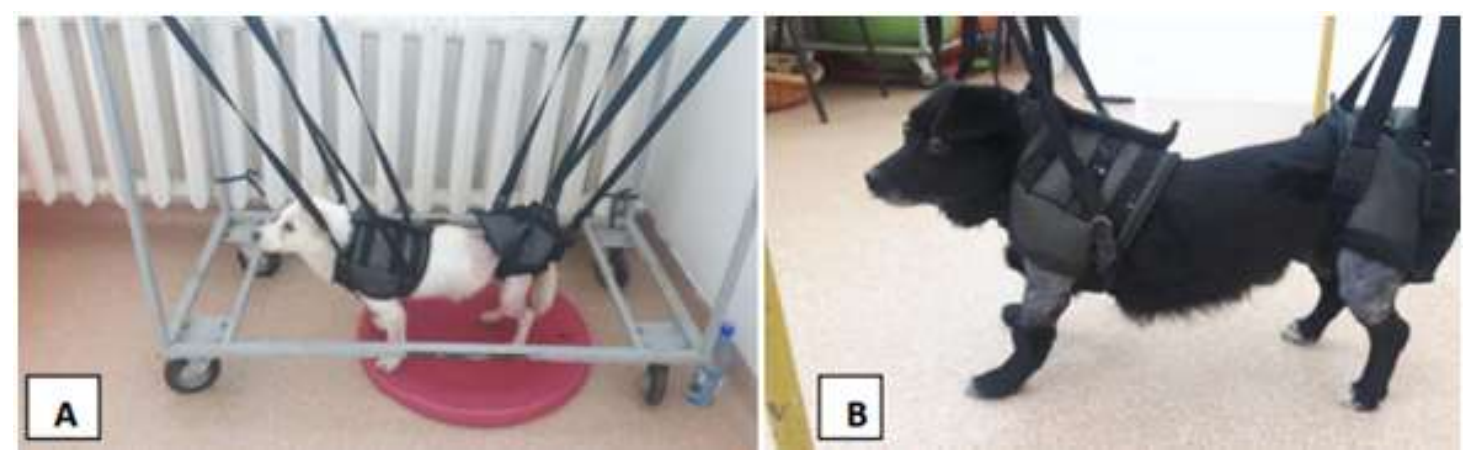

Figure 4. Adapting the standing position with the help of dog harnesses. A.Implementing the standing position using 


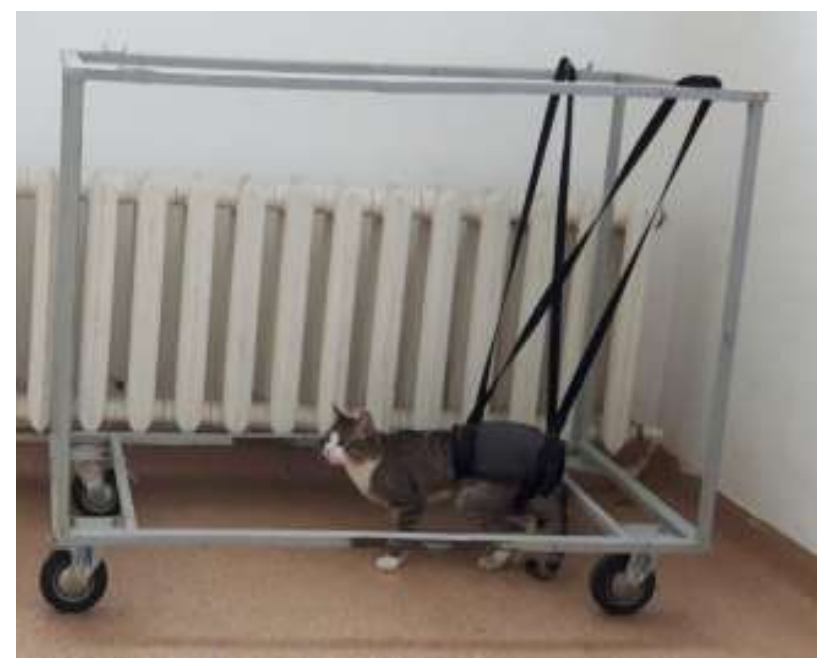

Figure 5. Adapting the standing position for cat using harnesses fixed to a wheeled device

3.1.3. Standing position actively supported with exercise rollers.

The active support of the animal can be achieved with the help of an exercise roller of appropriate size (Figure 6). However, this method requires the presence of two people, especially in cases of medium or large patients. Once the size of the roller is adjusted, the animal is placed in a standing position on it so that all four limbs touch the ground. This method is more effective than using an exercise ball considering its lack of stability. It is also preferred to be used as incompletely inflated rollers instead of the hard, fully inflated ones. Once the animal is placed on the roll, one person must provide support for the front limbs, and the second person will control the hind limbs. In this position the therapist can induce up-and-down or swaying movements. At the same time, as the patient develops endurance and muscular strength, the speed with which this exercise is performed can be increased for a better stimulation of balance and muscles [5, 6].



Figure 6. Inducing the standing position with the help of exercise rollers

is the next stage of the therapeutic process (Figure 7). At this stage, we can talk about a patient able to support themselves in the anatomical position, with the necessary strength and control. However, in some cases there is still weakness or incoordination, which can lead to the loss of balance and consequently will require assistance in regaining the standing position. The therapist places themselves on the patient's side, being prepared to support them in case they lose balance, intervening only if absolutely necessary. As the animal's condition improves, it increasingly seeks to move [10] 




Figure 7. Inducing the standing position independently, but with loss of balance

3.1.5. Recovering of proprioceptive sensitivity.

Once the patient acquires the ability to maintain the anatomical standing position without the intervention of the therapist, we move on to the stage which aims to regain the patient's balance (Figure 8). This way, we can mention the dynamic balance, a term that describes the patient's ability to maintain balance during motion. The techniques and methods used for this are applied on dry, non-slip surfaces, allowing the physiological locomotion in safe conditions, with low risk of falling.

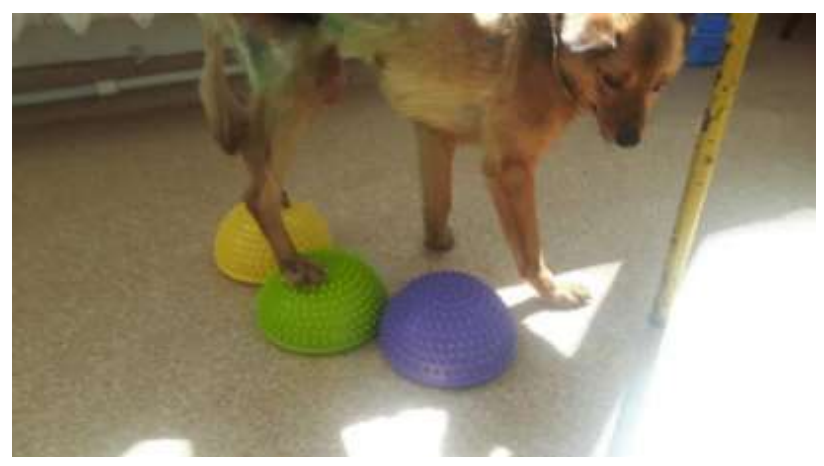

Figure 8. Regaining proprioception and balance

\subsubsection{Changing the center of gravity}

can be done by encouraging the animal with a treat or even a ball (Figure 9). The animal will carefully watch the treat or the ball, making up-and-down movements, as well as from right to left. Initially, the movements have a small amplitude, becoming more and more difficult during the therapeutic sessions. The key element in this stage is represented by the movement made with the head, this determining the patient to change their center of gravity using balance, strength and coordination. Playing with the ball, a more demanding activity, will be done by throwing the ball from a short distance, up and to the side. Initially, as a result of this exercise, the animal may show excessive enthusiasm that can lead to falls or bumps, requiring the presence of a person who is prepared to assist the animal. The therapist can test the animal's ability to regain balance by gently pushing the shoulders or hips, but also by removing the healthy limb from support. The change in the center of gravity can also be induced while strolling. While walking in a straight line, the therapist will gently push the animal to one side to encourage it to maintain its balance [4, 8]. 




Figure 9. Changing the center of gravity forcing the animal to adapt its center of gravity

\subsubsection{The balancing platform}

can be used for the forward and backward, sideways, diagonally and at $360^{\circ}$ swinging movements of the animal (Figure 10). In human medicine, the angle biomechanical platform system is used, being very similar to this equilibrium platform. It is important to mention that this biomechanical platform system can be used easily and with very good results in animals, with the aim of developing the proprioceptive capacity. In this sense, the front or the hind limbs are placed on the platform, while the opposite pair is on the ground (Figure 11). Ideally, one person should provide patient's support, and a second person should perform light and slow rocking movements of the platform. Thus, the animal is forced to change its center of gravity, training its proprioception [10].

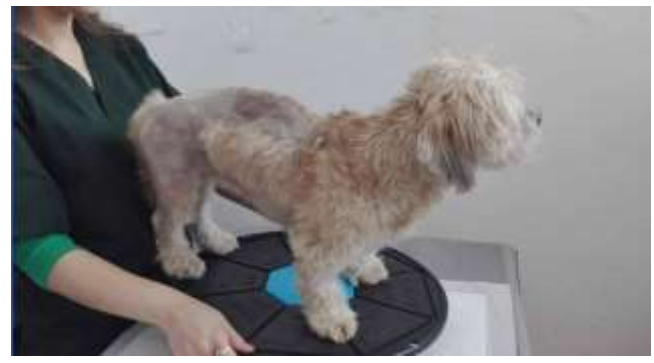

Figure 10. Balancing platform exercises


Figure 11. Exercises performed on the balance platform (A) and on the mattress (B)

\subsubsection{Exercise balls and rollers.}

For the development of balance, coordination and strength in animals, the use of balls and exercise rolls obtained for human use can be used (Figure 12). These devices have proven to be useful even in cases of common stretches. The exercises involve positioning the forelegs on the ball with the support of the therapist, forcing the animal to maintain 
static balance on the hind limbs. At the same time, the dynamic balance test can be performed by inducing forward-backward and lateral movements on the device, so that the hind limbs will be forced to maintain balance throughout these movements. When intervention is required on the neck and the anterior region of the body, the hind limbs are positioned above the device, forcing the front limbs to maintain balance and to support body weight both standing and during movements $[7,8]$.
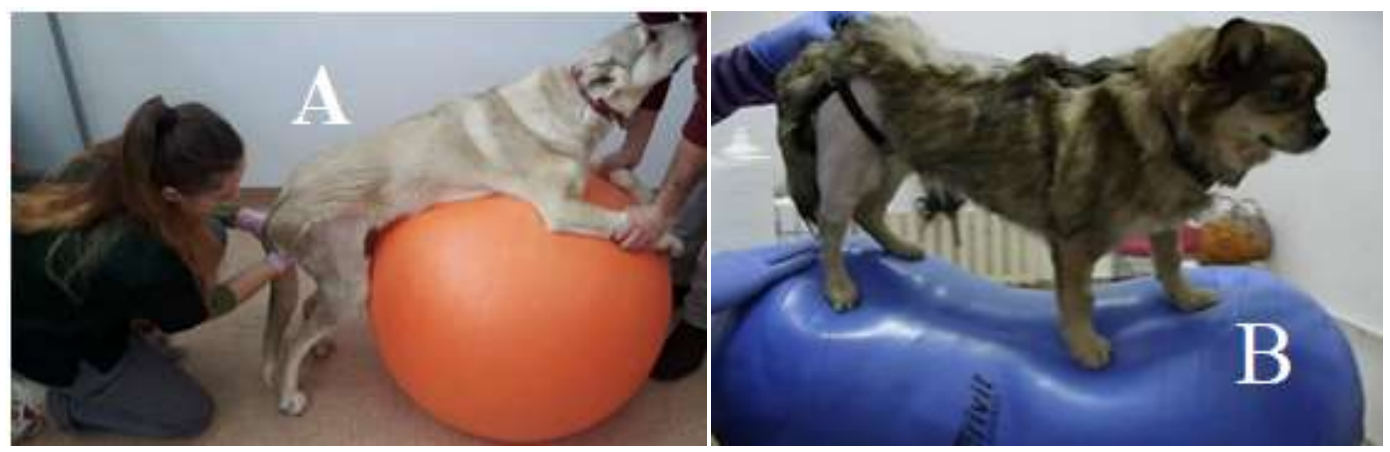

Figure 12. Exercises performed on the balance ball (A) and on the balance roller (B)

\subsubsection{Mobility exercises.}

If the patient does not have the ability to move on their own, a device designed to help in this regard may be used (Figure 13). The devices developed to facilitate the movement of patients are represented by: cardboard frames cut so that they can be adapted to the patient's height and weight (being very easy to handle and adjust); scarves and towels that can be attached to the metal frames aforementioned or can be handled directly by the therapist [4].

Initiating an effective workout is done with the help of straps, towels, harnesses or even trolleys. The therapist will choose the right device for the patient, after a prior assessment of their needs. The animal must be motivated and encouraged to move, even very slowly, but as independently as possible. The therapist can intervene if the animal needs its limbs to be placed in an anatomical position. It is also essential that the therapist and possibly the other people involved in the care and training of the animal are consistent, observing each patient's attempt to move too fast. If the animal tends to move too fast, there is a risk that it will avoid using the affected limb, leading to incorrect postures and moves: walking on three limbs, jumping or even crawling one limb [11].

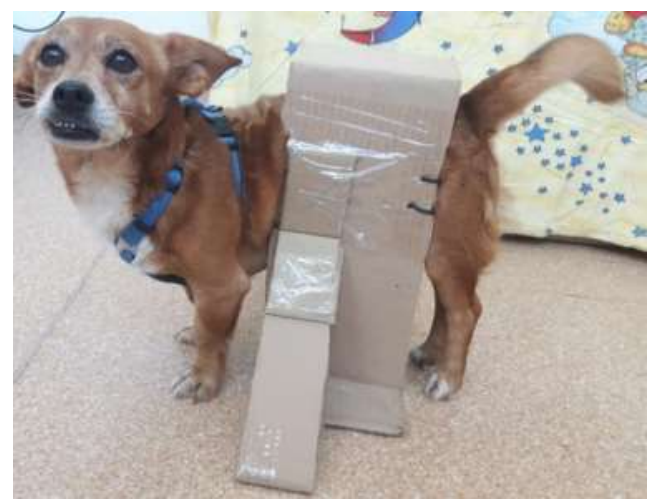

Figure 13. Supporting device made of cardboard, used to maintain the standing position

\subsubsection{Towels or scarves.}

Long towels can prove to be very useful in supporting animals that need help to maintain their standing position or to move (Figure 14). The great advantage of these devices is that they are cheap, and available to anyone. Also, a backup device is represented 
by scarves. Both options are useful, easy to find and affordable. For the support of the rear part of the body, the towel will be positioned around the abdominal region, right in front of the hind limbs. To ensure the optimal length for handling, the ends of the towel will be sewn with nylon strips. However, a disadvantage of using towels in large animals with urinary incontinence is that the towel, because of its location, will put pressure on the bladder, causing the urine to be excreted. However, this can be easily avoided by using a harness. Another disadvantage is that some towels may be uncomfortable for the therapist.

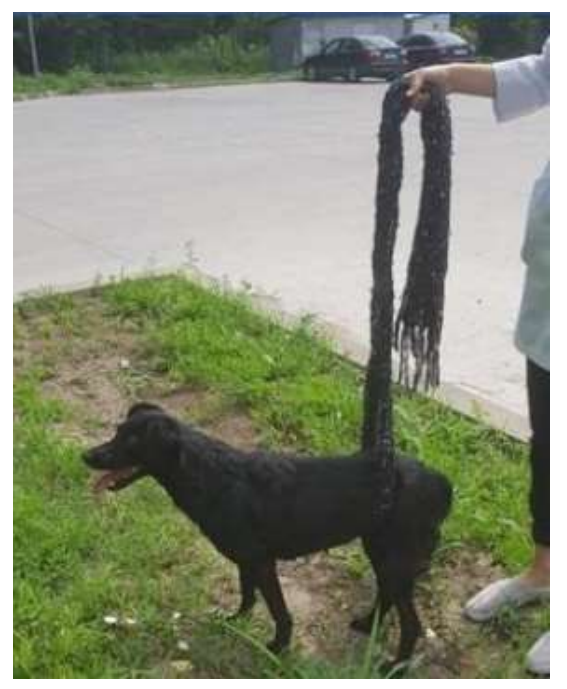

Figure 14. Inducing the standing position with a scarf

\subsubsection{Encouraging the support on the affected limb.}

A very useful way to encourage the animal to support the affected limb is to apply a plastic cap or a coin on the healthy limb and secure it with adhesive bands. Basically, by positioning the plastic cap on the pads of the healthy limb and then applying the adhesive band to fix the cap, the ideal element is obtained for encouraging the patient to support the affected limb. Although initial discomfort can be noticed, the device created will force the animal to use its affected limb correctly, both when resting and while walking, thus facilitating the return to physiological posture [10].

In most cases, the support on the affected limb is made even after removing the object from the unaffected limb (Figure 15). 


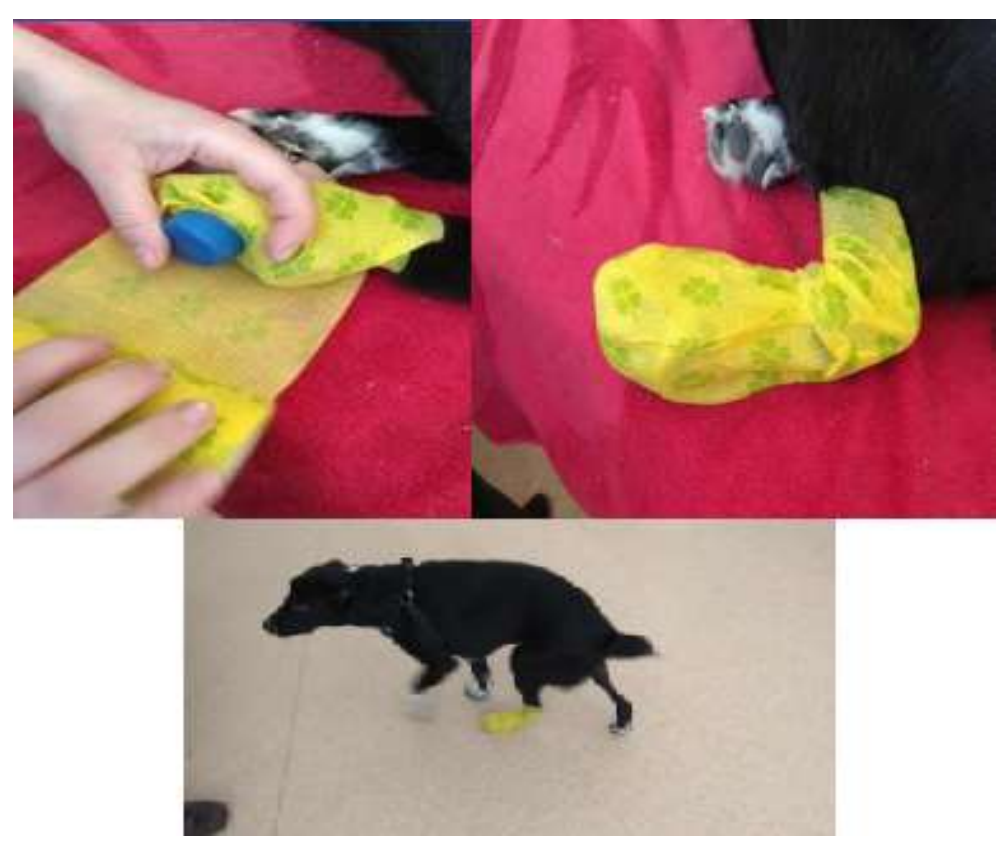

Figure 15. Applying a bottle cap to the healthy limb to encourage the use of the affected limb

\section{Conclusions}

We aimed to develop simple devices to mentain the standing position of paralyzed dogs and cats, accessible to anyone interested in physiotherapy or who takes care of patients with paralysis. The materials used to obtain these devices were easily obtained, at an affordable price, and subsequently they were rapidly assembled. They also allow paralyzed animal owners to develop their own similar variants so that they can contribute to the therapeutic protocol and to the complete healing of patients.

Author Contributions: Conceptualization, M.E.H., I.M. and G.S.; methodology, M.E.H., L.C.B. and E.V.S.; validation, M.E.H., L.C.B. and G.S.; formal analysis, C.S. and G.S.; investigation, M.E.H., I.M., M.G. and C.S.; writing-original draft preparation, M.E.H.; writing - review and editing, G.S.; supervision, G.S.; project administration, G.S.; funding acquisition, L.C.B. and E.V.S. All authors have read and agreed to the published version of the manuscript.

Funding: This work was supported by a grant of the Romanian Ministry of Research and Innovation, CCCDI-UEFISCDI, project number PN-III-P1- 1.2-PCCDI-2017-0239/60PCCDI 2018, within PNCDI III.

Institutional Review Board Statement: The study was conducted according to the guidelines of the Declaration of Helsinki. Ethical approval for the study was obtained from the Ethics Committee of the Faculty of Veterinary Medicine, University of Agricultural Sciences and Veterinary Medicine "Ion Ionescu de la Brad" from Iași (no. 430/05.05.2018).

Acknowledgments: The authors thank to Anca Maria Carter Timofte for reviewing and correcting the English version of the manuscript.

Conflicts of Interest: The authors declare no conflict of interest. The funders had no role in the design of the study; in the collection, analyses, or interpretation of data; in the writing of the manuscript, or in the decision to publish the results.

1.Piotti, P.; Karagiannis, C.; Satchell, L.; Michelazzi, M.; Albertini, M.; Alleva, E.; Pirrone, F., Use of the Milan Pet Quality of Life 
2.Levine, D.; Millis, D.L.; Myanatt, T., Effects of 3.3 MHz ultrasound on caudal thigh muscle temperature in dogs. Vet Surg 2001, 299

30:170-174. 300

3.Dragomir, M.F.; Pestean, C.P.; Melega, I.; Danciu, C.G.; Purdoiu, R.C.; Oana, L. Current Aspects Regarding the Clinical 301 Relevance of Electroacupuncture in Dogs with Spinal Cord Injury: A Literature Review. Animals 2021, 11, 219.

4.Henea, M.E., Applications of phisiotherapy in locomotory system diseases of companion carnivora (in Romanian), Ed. Ion Ionescu de 303 la Brad, Iași, Romania, 2020.

5.Brian, S., Physiotherapy in small animal practice. In practice 2008, 30:190-199.

6.Brian, $S_{.}$- Feline physiotherapy and rehabilitation. Principles and potential. JFMS 2012,14:622-632.

7.Darryl, M.; David, L., Canine rehabilitation and physical therapy, Saunders Elsevier, Knight, St Louis (MO), USA 2013.

8.Steiss, J.E., Canine rehabilitation. In: Braund's Clinical Neurology in Small Animal: Localization, diagnosis and treatment. Vite C.H., 308

Ed.; International Veterinary Information Service, Itacha, USA, 2010.

9.Steiss, J.E., Therapeutic ultrasound. In: Canine rehabilitation and physical therapy. Saunders Elsevier, St Louis, USA, 2004, pp 324- 310 336.

10. Levine, J.M.; Levine, G.J.; Boozer, L; Schatzberg, S.J.; Platt, S.R.; Kent, M.; Kerwin, S.C.; Fosgate, G.T., - Adverse effects and outcome associated with dexamethasone administration in dogs with acute thoracolumbar intervertebral disc herniation: 161 cases (2000-2006). JAVMA 2008, 232:411-417.

11. Levine, G.; Levine, J.; Budke, C.M.; Kerwin, S.C.; Au, J.; Vinayak, A.; Hettlich, B.F.; Slater, M.R., Description and repeatability a newly developed spinal cord scale for dogs. Prev Vet Med 2009, 89:121-127. 